\title{
Inverse backscattering for the acoustic equation
}

\author{
Plamen Stefanov* \\ Institute of Mathematics \\ Bulgarian Academy of Sciences \\ 1113 Sofia, Bulgaria
}

\author{
Gunther Uhlmann ${ }^{\dagger}$ \\ Department of Mathematics \\ University of Washington \\ Seattle, WA 98195, USA
}

\section{Introduction and statement of the results}

Consider the acoustic wave equation

$$
\left(\partial_{t}^{2}-c^{2}(x) \Delta\right) u=0, \quad(t, x) \in \mathbf{R} \times \mathbf{R}^{3}
$$

which describes the propagation of sound waves in an inhomogeneous medium with sound speed $c(x)$. We assume throughout the paper that $0<c(x), x \in \mathbf{R}^{3}$ and that for some $\rho>0$ we have

$$
c(x)=1 \quad \text { for }|x| \geq \rho .
$$

The scattering kernel measures, roughly speaking, the effect of the inhomogeneity on an incident plane wave of the form $\delta(t-x \cdot \theta)$ with $\theta \in S^{2}$. More precisely, assume that $c \in C^{2}\left(\mathbf{R}^{3}\right)$ and let $u(t, x, \theta)$ be the solution of the Cauchy problem

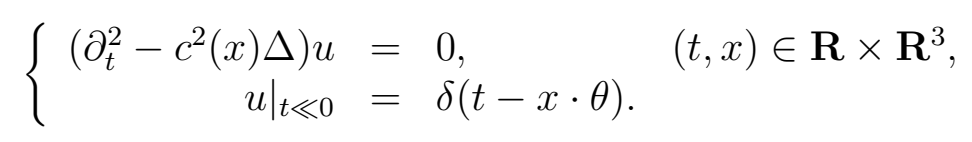

We have that

$$
u=\partial_{t}^{3} w
$$

where $w(t, x, \theta)$ solves

$$
\left\{\begin{array}{rl}
\left(\partial_{t}^{2}-c^{2}(x) \Delta\right) w & =0, \\
\left.w\right|_{t \ll 0} & =h_{2}(t-x \cdot \theta),
\end{array} \quad(t, x) \in \mathbf{R} \times \mathbf{R}^{3},\right.
$$

with $h_{2}(s)=s^{2} / 2$ for $s \geq 0$ and $h_{2}(s)=0$ otherwise. We write

$$
w=h_{2}(t-x \cdot \theta)+w_{\mathrm{sc}} .
$$

*Partly supported by BSF, Grant MM-407

${ }^{\dagger}$ Partly supported by NSF Grant DMS-9322619 and ONR Grant N00014-93-1-0295 
In the Lax-Phillips theory of scattering $[\mathrm{L}-\mathrm{P}]$ (see also $[\mathrm{C}-\mathrm{S}],[\mathrm{P}]$ ) the asymptotic wave profile $w_{\mathrm{sc}}^{\#}$ of $w_{\mathrm{sc}}$ is defined by

$$
w_{\mathrm{sc}}^{\#}(s, \omega, \theta)=\lim _{t \rightarrow \infty}(t+s) \partial_{t} w_{\mathrm{sc}}(t,(t+s) \omega, \theta),
$$

where the limit exists in $L^{2}\left(\mathbf{R}_{s} \times S_{\omega}^{2}\right)$ for any $\theta \in S^{2}$. Then the scattering kernel is given by

$$
S(s, \omega, \theta)=-\frac{1}{2 \pi} \partial_{s}^{3} w_{\mathrm{sc}}^{\#}(s, \omega, \theta) .
$$

We note that the scattering kernel $S$ is closely connected with the Schwartz kernel of the scattering operator $\mathcal{S}$. In fact, $S\left(s^{\prime}-s, \omega^{\prime}, \omega\right)$ is the Schwartz kernel of $\mathcal{R}(\mathcal{S}-I) \mathcal{R}^{-1}, \mathcal{R}$ being the Lax-Phillips translation representation [L-P] (see section 2).

The inverse backscattering problem consists in the determination of $c(x)$ from $S(s,-\theta, \theta)$. That is, roughly speaking, whether we can determine the sound speed by measuring the echoes produced by an incident plane wave in the direction $\theta$. In this paper we show that measuring the echoes is enough to recover the sound speed if it is a priori close to a constant.

Theorem 1.1 Let $S_{j}$ be the scattering kernel associated to the sound speed $c_{j}, j=1,2$ satisfying (1.2). Assume further that $c_{j} \in W^{9, \infty}\left(\mathbf{R}^{3}\right)$. There exists $\varepsilon>0$ such that if

$$
S_{1}(s,-\theta, \theta)=S_{2}(s,-\theta, \theta) \quad \text { for all } s \in \mathbf{R}, \theta \in S^{2}
$$

and if

$$
\left\|c_{j}-1\right\|_{W^{9, \infty}\left(\mathbf{R}^{3}\right)}<\varepsilon, \quad j=1,2,
$$

then we have $c_{1}=c_{2}$.

Guillemin proved in $[\mathrm{G}]$ that for the case considered here (and in more general situations) $\mathcal{S}$ is a Fourier integral operator and computed its symbol and canonical relation. In particular, $S(s,-\theta, \theta)$ makes sense and is a smooth function of $\theta$ with distributional values in the $s$-variable.

In the stationary approach to scattering one considers the formal Fourier transform of (1.1):

$$
\left(-\Delta+\lambda^{2}\left(1-c^{-2}(x)\right)-\lambda^{2}\right) v(x, \lambda)=0 .
$$

Notice that one can consider (1.4) as a Schrödinger equation with potential

$$
q(x)=\lambda^{2}\left(1-c^{-2}(x)\right) .
$$

However this is not very useful for the study of the inverse backscattering problem since we must consider high frequencies as well. The inverse scattering problem at a fixed energy has been solved in dimension $n \geq 3$ by Novikov [N]. This problem is in fact closely related to the inverse problem of determining a potential $q$ from its associated Dirichlet to Neumann map. The latter problem was solved in [S-U]. For an account of this relationship see for instance [U]. 
Given any $\theta \in S^{2}$ there are solutions of (1.4) of the form

$$
v(x, \theta, \lambda)=e^{i \lambda x \cdot \theta}+\frac{e^{i \lambda|x|}}{|x|} a(\lambda, \omega, \theta)+o\left(|x|^{-1}\right), \quad \text { as }|x| \rightarrow \infty,
$$

where $\omega=x /|x|$. The function $a$ is called the scattering amplitude. The relation between $a$ and $S$ is very simple

$$
\frac{i \lambda}{2 \pi} a(\lambda, \omega, \theta)=\int e^{-i s \lambda} S(s, \omega, \theta) d s .
$$

Theorem 1.1 has therefore as immediate corollary:

Theorem 1.2 Let $c_{j}, j=1,2$ be as in Theorem 1.1. Let $a_{j}$ denote the scattering amplitude associated to $c_{j}, j=1,2$. There exists $\varepsilon>0$ such that if

$$
a_{1}(\lambda,-\theta, \theta)=a_{2}(\lambda,-\theta, \theta)
$$

and if

$$
\left\|c_{j}-1\right\|_{W^{9, \infty}\left(\mathbf{R}^{3}\right)}<\varepsilon, \quad j=1,2,
$$

then $c_{1}=c_{2}$.

The high frequency asymptotics of the scattering amplitude has been considered in $[\mathrm{G}]$ and $[\mathrm{V}]$. We do not know of any result for the inverse backscattering problem for the acoustic equation. The inverse backscattering problem for the Schrödinger equation has been studied in the papers $[\mathrm{E}-\mathrm{R}]$, [St II].

The structure of the paper is as follows. In section 2 we consider some preliminaries and prove Proposition 2.1 which gives a relation between $S_{1}-S_{2}$ and $c_{1}^{-2}-c_{2}^{-2}$. In section 3 we construct the singular solution of (1.3). In section 4 we prove Theorem 1.1 by combining the results of section 3 and inverting a generalized Radon transform.

\section{Preliminaries}

In this section we introduce the scattering kernel $S(s, \omega, \theta)$ and in Proposition 2.1 we prove a formula for the difference $S_{1}-S_{2}$, where $S_{j}, j=1,2$ are related to two sound speeds $c_{j} \in C^{2}$ satisfying (1.2). A formula of a similar type related to a potential perturbation of the wave equation was first obtained in [St I].

The natural energy space for equation (1.1) is the completion $\mathcal{H}$ of $C_{0}^{\infty}\left(\mathbf{R}^{3}\right) \times C_{0}^{\infty}\left(\mathbf{R}^{3}\right)$ with respect to the energy norm

$$
\|f\|_{\mathcal{H}}^{2}=\frac{1}{2} \int\left(\left|\nabla f_{1}\right|^{2}+c^{-2}(x)\left|f_{2}\right|^{2}\right) d x, \quad f=\left[f_{1}, f_{2}\right] .
$$

Throughout this paper we will denote two-dimensional vector functions ${ }^{t}\left(f_{1}, f_{2}\right)$ by $\left[f_{1}, f_{2}\right]$. Then $\mathcal{H}$ is a Hilbert space and equation (1.1) is equivalent to

$$
\partial_{t} u=-i A u, \quad \text { with } \quad u=\left[u_{1}, u_{2}\right], \quad A=i\left(\begin{array}{cc}
0 & I \\
c^{2} \Delta & 0
\end{array}\right),
$$


i.e. if $u$ solves $(2.1)$, then $u_{2}=\partial_{t} u_{1},\left(\partial_{t}^{2}-c^{2} \Delta\right) u_{1}=0$. Here $I$ stands for the identity map. It is easy to see that $A$ extends to a self-adjoint operator in $\mathcal{H}$, therefore the solution to (2.1) is given by $u=e^{-i t A} f=: U(t) f$, where $f=\left.u\right|_{t=0}$. By Stone's theorem $U(t)$ forms a strongly continuous group of unitary operators in $\mathcal{H}$. Setting $c=1$, we get the unperturbed group $U_{0}(t)$ in $\mathcal{H}_{0}$ related to the unperturbed wave equation $\left(\partial_{t}^{2}-\Delta\right) u=0$. The scattering operator $\mathcal{S}$ is then defined by $\mathcal{S}=W_{-}^{-1} W_{+}$, where the wave operators $W_{ \pm}$are defined as the strong limits $W_{ \pm}=\mathrm{s}_{-} \lim _{t \rightarrow \pm} U(t) U_{0}(-t)$. It is well known that the wave operators exist as bounded operators and moreover, $\mathcal{S}$ is also well defined as a bounded operator in $\mathcal{H}_{0}$ [L-P], [R-S].

As in the Introduction, we consider the scattering solution $u(t, x, \theta)$ as the solution to the following Cauchy problem

$$
\left\{\begin{array}{rlrl}
\left(\partial_{t}^{2}-c^{2} \Delta\right) u & =0 & & \text { in } \mathbf{R}_{t} \times \mathbf{R}_{x}^{3}, \\
\left.u\right|_{t \ll 0} & =\delta(t-x \cdot \theta) . &
\end{array}\right.
$$

Here $\theta \in S^{2}$ is a parameter giving the direction of the incident plane wave in (2.2). The initial condition above can be replaced by $\left.u\right|_{t=-\rho}=\delta(-\rho-x \cdot \theta),\left.u_{t}\right|_{t=-\rho}=\delta^{\prime}(-\rho-x \cdot \theta)$. The standard way of constructing a solution of (2.2) is the following. Set $h_{j}(t)=t^{j} / j$ ! for $t \geq 0$ and $h_{j}(t)=0$ otherwise. Then $h_{j}^{\prime}=h_{j-1}, j \geq 1$ and $h_{0}$ is the Heaviside function. If we replace the Dirac delta function $\delta$ in $(2.2)$ by $h_{2}$, we get initial data $\left[h_{2}(-\rho-x \cdot \theta), h_{1}(-\rho-x \cdot \theta)\right]$ for $t=-\rho$, that belong locally to $\mathcal{H}$ and even to $D(A)$. As in the Introduction, consider the problem

$$
\left\{\begin{array}{rlrl}
\left(\partial_{t}^{2}-c^{2} \Delta\right) w & =0 & & \text { in } \mathbf{R}_{t} \times \mathbf{R}_{x}^{3}, \\
\left.w\right|_{t \ll 0} & =h_{2}(t-x \cdot \theta) . &
\end{array}\right.
$$

Then $w=h_{2}(t-x \cdot \theta)+w_{\mathrm{sc}}$, where $\left(\partial_{t}^{2}-c^{2} \Delta\right) w_{\mathrm{sc}}=-\left(1-c^{2}\right) h_{0}(t-x \cdot \theta)$ and $\left.w_{\mathrm{sc}}\right|_{t \ll 0}=0$. Therefore,

$$
\left[w_{\mathrm{sc}}, \partial_{t} w_{\mathrm{sc}}\right]=-\int_{-\infty}^{t} U(t-s)\left(1-c^{2}\right)\left[0, h_{0}(s-x \cdot \theta)\right] d s .
$$

Here $1-c^{2}$ has compact support thus $\left(1-c^{2}\right)\left[0, h_{0}(s-x \cdot \theta)\right] \in \mathcal{H}$. Having constructed a solution to $(2.3)$ we can now solve $(2.2)$ by setting

$$
u(t, x, \theta)=\partial_{t}^{3} w(t, x, \theta) .
$$

Following Lax-Phillips [L-P] (see also [C-S]), as in the Introduction we define the asymptotic wave profile $w_{\mathrm{sc}}^{\#}$ of $w_{\mathrm{sc}}$ by

$$
w_{\mathrm{sc}}^{\#}(s, \omega, \theta)=\lim _{t \rightarrow \infty}(t+s) \partial_{t} w_{\mathrm{sc}}(t,(t+s) \omega, \theta) .
$$

The limit exists in $L^{2}\left(\mathbf{R}_{s} \times S_{\omega}^{2}\right)$ for any $\theta[\mathrm{L}-\mathrm{P}],[\mathrm{C}-\mathrm{S}]$. Then we define the scattering kernel $S$ by

$$
S(s, \omega, \theta)=-\frac{1}{2 \pi} \partial_{s}^{3} w_{\mathrm{sc}}^{\#}(s, \omega, \theta) .
$$

In some sense $S$ satisfies the asymptotics

$$
\partial_{t} u(t, x, \theta)=\delta^{\prime}(t-x \cdot \theta)-\frac{2 \pi}{|x|} S\left(|x|-t, \frac{x}{|x|}, \theta\right)+o\left(\frac{1}{|x|}\right), \quad \text { as } t,|x| \rightarrow \infty .
$$


The formula above is a time-dependent analogue of the definition (1.5) of the scattering amplitude via the asymptotics of the solution $v$ of the Lipmann-Schwinger equation for large $x$.

It turns out that $S$ is closely related to the distribution kernel of the scattering operator $\mathcal{S}$. Denote by $(R f)(s, \omega)=\int f(x) \delta(s-x \cdot \omega) d x$ the Radon transform of $f$ and consider the operator $\mathcal{R}$ (the Lax and Phillips translation representation) defined by $\mathcal{R}\left[f_{1}, f_{2}\right]=$ $\frac{1}{4 \pi}\left(-\partial_{s}^{2} R f_{1}+\partial_{s} R f_{2}\right)$. Then $\mathcal{R}$ is a unitary map $\mathcal{R}: \mathcal{H}_{0} \rightarrow L^{2}\left(\mathbf{R} \times S^{2}\right)$. A well known fact form the Lax and Phillips theory is that $S\left(s^{\prime}-s, w^{\prime}, w\right)$ is the Schwartz kernel of $\mathcal{R}(\mathcal{S}-I) \mathcal{R}^{-1}$ (see $[\mathrm{L}-\mathrm{P}],[\mathrm{C}-\mathrm{S}],[\mathrm{P}]$ ), i.e. in distribution sense we have

$$
\left(\mathcal{R}(\mathcal{S}-I) \mathcal{R}^{-1} k\right)\left(s^{\prime}, \omega^{\prime}\right)=\int_{\mathbf{R} \times S^{2}} S\left(s^{\prime}-s, \omega^{\prime}, \omega\right) k(s, \omega) d s d \omega .
$$

Next we will derive a formula for $S_{1}-S_{2}$, where $S_{j}$ is related to $c_{j}, j=1,2$. Let us first notice that $(2 \pi)^{-1}\left[u( \pm t \pm s, x, \pm \theta), \partial_{t} u( \pm t \pm s, x, \pm \theta)\right]$ is the distribution kernel of $U(t) W_{ \pm} \mathcal{R}^{-1}$, i.e. for any $k \in C_{0}^{\infty}\left(\mathbf{R} \times S^{2}\right)$ in distribution sense we have

$$
U(t) W_{ \pm} \mathcal{R}^{-1} k=\frac{1}{2 \pi} \int_{\mathbf{R} \times S^{2}}\left[u( \pm t \pm s, x, \pm \theta), \partial_{t} u( \pm t \pm s, x, \pm \theta)\right] k(s, \theta) d s d \theta .
$$

Indeed, denote $f=\mathcal{R}^{-1} k$ and consider $W_{+}$. Then $U(t) W_{+} \mathcal{R}^{-1} k=U(t+T) U_{0}(-T) f$ for some fixed $T>0$ depending on $\operatorname{supp} k$. Denote $\left[v, \partial_{t} v\right]=U(t+T) U_{0}(-T) f$ and denote also the right-hand side of (2.9) by $\left[\tilde{v}, \partial_{t} \tilde{v}\right]$. Both $v$ and $\tilde{v}$ solve (1.1). Next, for $t<-T$ we have $\left[v, \partial_{t} v\right]=U_{0}(t) f$. On the other hand, for $t \ll 0$ we get for $\tilde{v}$

$$
\left[\tilde{v}, \partial_{t} \tilde{v}\right]=\frac{1}{2 \pi} \int_{\mathbf{R} \times S^{2}}\left[\delta(t+s-x \cdot \theta), \delta^{\prime}(t+s-x \cdot \theta)\right] k(s, \theta) d s d \theta=U_{0}(t) f
$$

by the inversion formula for $\mathcal{R}$ (see [L-P]). Therefore, $v$ and $\tilde{v}$ have the same initial data and must coincide. This proves (2.9) for $W_{+}$. The proof for $W_{-}$is similar.

Proposition 2.1 Let $S_{j}(s, \omega, \theta)$ be the scattering kernel related to $c_{j}(x) \in C^{2}, j=1,2$. Then

$$
\left(S_{1}-S_{2}\right)(s, \omega, \theta)=\frac{1}{8 \pi^{2}} \partial_{s}^{3} \iint\left(c_{1}^{-2}-c_{2}^{-2}\right) u_{1}(t, x, \theta) u_{2}(-s-t, x,-\omega) d t d x,
$$

where $u_{j}$ are the scattering solutions related to $c_{j}, j=1,2$ and the integral is to be considered in distribution sense.

Proof. Denote by $U_{j}(t), j=1,2$ the propagators related to $c_{j}$. Consider the function $F(t)=$ $U_{2}(T+t) U_{1}(-t+T) f, f \in D\left(A_{1}\right)=D\left(A_{2}\right)$. Then $F^{\prime}(t)=-i U_{2}(T+t)\left(A_{2}-A_{1}\right) U_{1}(-t+T)$ and from $F(T)-F(-T)=\int_{-T}^{T} F^{\prime}(t) d t$ we get

$$
\left(U_{2}(2 T)-U_{1}(2 T)\right) f=\int_{-T}^{T} U_{2}(T+t) Q U_{1}(-t+T) f d t,
$$

where

$$
Q=\left(\begin{array}{cc}
0 & 0 \\
\left(c_{2}^{2}-c_{1}^{2}\right) \Delta & 0
\end{array}\right)
$$


Next, choose two functions $k, l \in C_{0}^{\infty}\left(\mathbf{R} \times S^{2}\right)$ and set $f=\mathcal{R}^{-1} k, g=\mathcal{R}^{-1} l$. Then by using standard arguments from the Lax-Phillips theory we get that

$$
\left(\mathcal{S}_{j} f, g\right)_{\mathcal{H}_{0}}=\left(U_{0}(-T) U_{j}(2 T) U_{0}(-T) f, g\right)_{\mathcal{H}_{0}}
$$

with some large $T>0$ depending on $\operatorname{supp} k, \operatorname{supp} l$. Therefore, by (2.10)

$$
\begin{aligned}
\left(\left(\mathcal{S}_{2}-\mathcal{S}_{1}\right) f, g\right)_{\mathcal{H}_{0}} & =\int_{-T}^{T}\left(U_{0}(-T) U_{2}(T+t) Q U_{1}(-t+T) U_{0}(-T) f, g\right)_{\mathcal{H}_{0}} d t \\
& =\int_{-T}^{T}\left(Q U_{1}(-t+T) U_{0}(-T) f, U_{2}(-t-T) U_{0}(T) g\right)_{\mathcal{H}_{2}} d t
\end{aligned}
$$

Here $\mathcal{H}_{j}, j=0,1,2$ are related to $c_{0}=1, c_{1}$, and $c_{2}$ respectively. Next, note that $U_{1}(-t+T) U_{0}(-T) f=U_{1}(-t) W_{+}^{(1)} f=U_{1}(-t) W_{+}^{(1)} \mathcal{R}^{-1} k$. Similarly, $U_{2}(-t-T) U_{0}(T) g=$ $U_{2}(-t) W_{-}^{(2)} \mathcal{R}^{-1} l$. Using (2.9), we get from $(2.11)$

$$
\begin{aligned}
\left(\left(\mathcal{S}_{2}-\mathcal{S}_{1}\right) f, g\right)_{\mathcal{H}_{0}}= & \frac{1}{8 \pi^{2}} \int_{-T}^{T} \int \ldots \int\left(c_{2}^{2}-c_{1}^{2}\right)\left(\Delta u_{1}\right)\left(-t+s_{1}, x, \theta_{1}\right) \partial_{t} u_{2}\left(t-s_{2}, x,-\theta_{2}\right) \\
& \times k\left(s_{1}, \theta_{1}\right) l\left(s_{2}, \theta_{2}\right) c_{2}^{-2} d s_{1} d \theta_{1} d s_{2} d \theta_{2} d x d t \\
=\frac{1}{8 \pi^{2}} \int_{-T}^{T} \int & \ldots \int\left(c_{1}^{-2}-c_{2}^{-2}\right) \partial_{s_{1}}^{2} u_{1}\left(-t+s_{1}, x, \theta_{1}\right) \partial_{t} u_{2}\left(t-s_{2}, x,-\theta_{2}\right) \\
& \times k\left(s_{1}, \theta_{1}\right) l\left(s_{2}, \theta_{2}\right) d s_{1} d \theta_{1} d s_{2} d \theta_{2} d x d t
\end{aligned}
$$

Clearly, the integrand above vanishes for $|t|>T$, so we may integrate in $t$ over the whole real line. According to (2.8),

$$
\left(\left(\mathcal{S}_{2}-\mathcal{S}_{1}\right) f, g\right)_{\mathcal{H}_{0}}=\int_{\left[\mathbf{R} \times S^{2}\right]^{2}}\left(S_{2}-S_{1}\right)\left(s_{2}-s_{1}, \theta_{2}, \theta_{1}\right) k\left(s_{1}, \theta_{1}\right) l\left(s_{2}, \theta_{2}\right) d s_{1} d \theta_{1} d s_{2} d \theta_{2}
$$

Comparing (2.12) and (2.13), we conclude that

$$
\left(S_{1}-S_{2}\right)\left(s_{2}-s_{1}, \theta_{2}, \theta_{1}\right)=\frac{1}{8 \pi^{2}} \iint\left(c_{1}^{-2}-c_{2}^{-2}\right) \partial_{s_{1}}^{2} u_{1}\left(-t+s_{1}, x, \theta_{1}\right) \partial_{t} u_{2}\left(t-s_{2}, x,-\theta_{2}\right) d x d t
$$

The right-hand side above as a function of $s_{1}, s_{2}$ depends merely on $s_{2}-s_{1}$ and setting $s=s_{2}-s_{1}, \tilde{t}=-t+s_{1}$ we complete the proof of the proposition.

\section{Singular decomposition of the scattering solution}

In this section we prove that the scattering solution $u(t, x, \theta)$ admits a singular decomposition of the type $u(t, x, \theta)=\alpha(x, \theta) \delta(t-\phi(x, \theta))+\beta(x, \theta) h_{0}(t-\phi(x, \theta))+r(t, x, \theta)$, where $\phi$ is a suitable phase function and the remainder $r(t, \cdot, \theta)$ belongs to $H^{1} \cap L^{\infty}, \partial_{t} r \in L^{2}$. Such decompositions are in principle known for that kind of problems (see e.g. [V] for a high frequency asymptotics of the solution $v$ of (1.4) given by (1.5)). Our goal here is to prove estimates on the remainder which are uniform in $c(x)$ under the assumption of a finite smoothness of $c$. As in Theorem 1.1 we assume that $c$ is close to $c=1$ in the $W^{m, \infty}$ topology 
for some $m$. It turns out that in our proof we need estimates on the remainder for $t$ belonging to a finite interval only. This fact simplifies considerably our analysis. On the other hand, in principle one could obtain estimates on the remainder for large $t$ which are also uniform in $c$. This is related to the problem of finding estimates of the remainder in the high-frequency asymptotics of the solution $v$ of (1.4) defined in (1.5) (see [V]) which are uniform in $c$ or finding estimates on the resolvent of $c^{2} \Delta+\lambda^{2}$. The latter problems are more delicate ones. In fact one of the main reasons for working with time dependent methods is the advantage we get by dealing with bounded t's only.

We start with analysis of the phase function $\phi$ related to (1.1). We define $\phi(x, \theta)$ as the solution to the eikonal equation

$$
\left\{\begin{aligned}
(\nabla \phi)^{2} & =c^{-2}(x) \\
\left.\phi\right|_{x \cdot \theta \ll 0} & =x \cdot \theta
\end{aligned}\right.
$$

Throughout this section we assume that $c$ satisfies (1.2) and that

$$
\|c-1\|_{W^{m, \infty}}<\varepsilon
$$

with some $\varepsilon>0$ and $m \geq 2$. We need to solve (3.1) in $B_{\rho}$. Fix $\theta \in S^{2}$. We may assume that $\theta={ }^{t}(1,0,0)$. Then $(3.1)$ can be rewritten as

$$
\left\{\begin{aligned}
(\nabla \phi)^{2} & =c^{-2}(x) \\
\left.\phi\right|_{x_{1}=-\rho} & =-\rho \\
\left.\partial_{x_{1}} \phi\right|_{x_{1}=-\rho} & =1
\end{aligned}\right.
$$

The Hamiltonian system associated with (3.3) is

$$
\left\{\begin{aligned}
\frac{d}{d s} x & =2 \xi, & \frac{d}{d s} \xi & =\nabla c^{-2}, \\
\left.x\right|_{s=0} & ={ }^{t}(-\rho, \eta), & \left.\xi\right|_{s=0} & ={ }^{t}(1,0,0),
\end{aligned}\right.
$$

Notice that the solution to (3.4) in the case $c=1$ is $x={ }^{t}(2 s-\rho, \eta), \xi={ }^{t}(1,0,0)$. On the other hand, for general $c(x)$ the solution of (3.4) exists for any $s$ (see $[\mathrm{V}]$ ).

Lemma 3.1 Fix $a>0$. Then there exists $C>0$ such that for the solution $x=x(s, \eta)$, $\xi=\xi(s, \eta)$ of (3.4) we have

$$
\left\|x-{ }^{t}(2 s-\rho, \eta)\right\|_{W^{m, \infty}\left([0, a] \times \mathbf{R}^{2}\right)}+\left\|\xi-{ }^{t}(1,0,0)\right\|_{W^{m, \infty}\left([0, a] \times \mathbf{R}^{2}\right)} \leq C \varepsilon .
$$

The proof of the lemma is based on a comparison theorem for ODE and will be omitted here.

In particular, Lemma 3.1 implies that under the smallness assumption (3.2) the Hamiltonian flow is non-trapping for small $\varepsilon$, more precisely, $x(s, \eta) \notin B_{\rho}=\{x ;|x|<\rho\}$ for $s>a$ with some $a>0$. Moreover, the mapping ${ }^{t}(s, \eta) \mapsto x(s, \eta)$ is a $W^{m, \infty}$-diffeomorphism on $[0, a] \times\left\{\eta \in \mathbf{R}^{2} ;|\eta| \leq 2 \rho\right\}$ and its range covers $B_{\rho}$ provided that $\varepsilon$ is small enough. We will need in fact to work in a larger domain, so let us assume that $\varepsilon$ and $a$ are such that 
${ }^{t}(s, \eta) \mapsto x(s, \eta)$ maps $[0, a] \times\left\{\eta \in \mathbf{R}^{2} ;|\eta| \leq 5 \rho\right\}$ into a compact covering $B_{4 \rho}$. The phase function $\phi$ solving (3.3) is defined in $B_{4 \rho}$ by (see [V])

$$
\phi=-\rho+2 \int c^{-2}(x) d s
$$

where the integration is taken over the shortest characteristics $x=x(s, \eta)$ joining the plane $x_{1}=-\rho$ and $x$. The change of coordinates $x \mapsto{ }^{t}(s, \eta)$ is $\varepsilon$-close to $x={ }^{t}(2 s-\rho, \eta)$ in $W^{m, \infty}$, which easily implies that $\phi$ must be close to $\phi=x_{1}$. So far $\theta$ was fixed. One can also examine easily the dependence of $\phi$ on $\theta \in S^{2}$. Thus we get

Lemma 3.2 Assume that (3.2) holds with $\varepsilon>0$ sufficiently small. Then there exists $C_{0}>0$ such that

$$
\|\phi(x, \theta)-x \cdot \theta\|_{W^{m, \infty}\left(B_{4 \rho} \times S^{2}\right)} \leq C_{0} \varepsilon .
$$

Now we are ready to prove the principal result of this section about the scattering solution $u(t, x, \theta)$ introduced in $(2.2)$. Denote

$$
T=\rho+C_{0} \varepsilon,
$$

where $C_{0}$ is the constant in Lemma 3.2. Note that $\max \left\{|\phi(x, \theta)| ; x \in B_{\rho}, \theta \in S^{2}\right\} \leq T$.

Proposition 3.1 Assume that (3.2) holds with $m \geq 9$ and $\varepsilon>0$ sufficiently small. Then there exists a constant $C>0$, such that for $|t|<3 T$, and for any $\theta \in S^{2}$ we have

$$
u(t, x, \theta)=\alpha(x, \theta) \delta(t-\phi(x, \theta))+\beta(x, \theta) h_{0}(t-\phi(x, \theta))+r(t, x, \theta),
$$

where

$$
\|\alpha-1\|_{W^{m-2, \infty}\left(B_{4 \rho} \times S^{2}\right)} \leq C \varepsilon, \quad|\beta(x, \theta)| \leq C \varepsilon,
$$

and

$$
\|r(t, \cdot, \theta)\|_{L^{\infty}}+\left\|\partial_{t} r(t, \cdot, \theta)\right\|_{L^{2}} \leq C \varepsilon
$$

Proof. Let us look for $u$ of the form

$u(t, x, \theta)=\alpha(x, \theta) \delta(t-\phi(x, \theta))+\beta(x, \theta) h_{0}(t-\phi(x, \theta))+\gamma(x, \theta) h_{1}(t-\phi(x, \theta))+\tilde{r}(t, x, \theta)$.

Then $\alpha=1+\tilde{\alpha}, \beta, \gamma$ solve the transport equations

$$
\begin{array}{ll}
(2 \nabla \phi \cdot \nabla+\Delta \phi) \tilde{\alpha}=-\Delta \phi, & \left.\tilde{\alpha}\right|_{x \cdot \theta=-\rho}=0 \\
(2 \nabla \phi \cdot \nabla+\Delta \phi) \beta=\Delta \alpha, & \left.\beta\right|_{x \cdot \theta=-\rho}=0 \\
(2 \nabla \phi \cdot \nabla+\Delta \phi) \gamma=\Delta \beta, & \left.\gamma\right|_{x \cdot \theta=-\rho}=0
\end{array}
$$

while $\tilde{r}$ solves

$$
\left(c^{-2} \partial_{t}^{2}-\Delta\right) \tilde{r}=(\Delta \gamma) h_{1}(t-\phi),\left.\quad \tilde{r}\right|_{t \ll 0}=0 .
$$

Note that we need to solve (3.8) - (3.10) in the compact $x \cdot \theta \geq-\rho, \phi(x, \theta) \leq 3 T,|\eta|<\rho$ $(\eta=\eta(x)$ is determined by $x=x(s, \eta))$ and for $\varepsilon$ sufficiently small this compact is contained 
in $B_{4 \rho}$, where $\phi$ is well defined. The first equation (3.8) can be solved in $B_{4 \rho}$ and (3.6) follows directly from Lemma 3.2. The estimate (3.6) for $\alpha$ follows easily from Lemma 3.1 and Lemma 3.2. Next, since $\Delta \alpha=O(\varepsilon)$, we get (if $m \geq 4$ ) (3.6) for $\beta$ as well. Similarly, if $m \geq 6$, then $|\gamma|=O(\varepsilon)$ as well. Finally, for $\tilde{r}$ we get by (3.11)

$$
\left[\tilde{r}, \partial_{t} \tilde{r}\right]=\int_{-\rho}^{t} U(t-s)\left[0,(\Delta \gamma) h_{1}(s-\phi)\right] d s .
$$

We get as above that $(\Delta \gamma) h_{1}(s-\phi)$ is supported in $B_{4 \rho}$ for $-\rho \leq s \leq t,|t|<3 T$ and moreover $\left\|\left[0,(\Delta \gamma) h_{1}(s-\phi)\right]\right\|_{\mathcal{H}} \leq C \varepsilon$ (if $\left.m \geq 8\right)$. Note that the norm in $\mathcal{H}$ depends on $c(x)$, but is uniformly bounded when $c$ satisfies (3.2) with $\varepsilon<1$. So we get

$$
\left\|\left[\tilde{r}, \partial_{t} \tilde{r}\right]\right\|_{\mathcal{H}} \leq C(t+\rho) \varepsilon, \quad-\rho \leq t \leq T
$$

(and $\tilde{r}=0$ for $t<-\rho$ ). Next, $\left[\tilde{r}, \partial_{t} \tilde{r}\right] \in D(A)$ and

$$
\begin{aligned}
A\left[\tilde{r}, \partial_{t} \tilde{r}\right]=\left[\partial_{t} \tilde{r}, c^{2} \Delta \tilde{r}\right] & =\int_{-\rho}^{t} U(t-s) A\left[0,(\Delta \gamma) h_{1}(s-\phi)\right] d s \\
& =\int_{-\rho}^{t} U(t-s)\left[(\Delta \gamma) h_{1}(s-\phi), 0\right] d s
\end{aligned}
$$

Since $\left\|\left[(\Delta \gamma) h_{1}(s-\phi), 0\right]\right\|_{\mathcal{H}}=O(\varepsilon)$ (here we need $m=9$ ), we get as above that

$$
\left\|\left[\partial_{t} \tilde{r}, c^{2} \Delta \tilde{r}\right]\right\|_{\mathcal{H}} \leq C(t+\rho) \varepsilon, \quad-\rho \leq t \leq T .
$$

By (3.12) and (3.13),

$$
\|\nabla \tilde{r}\|+\|\Delta \tilde{r}\|+\left\|\partial_{t} \tilde{r}\right\|+\left\|\nabla \partial_{t} \tilde{r}\right\| \leq C \varepsilon,
$$

where $\|\cdot\|=\|\cdot\|_{L^{2}}$. Moreover, $\tilde{r}$ is compactly supported (uniformly in $\varepsilon<1,|t|<3 T$ ) because of the finite speed of propagation for (1.1). Therefore, by the Poincaré inequality (see e.g. [L-P]), we get $\|\tilde{r}\|=O(\varepsilon)$ as well. Thus,

$$
\|\tilde{r}\|_{H^{2}}+\left\|\partial_{t} \tilde{r}\right\|_{H^{1}} \leq C \varepsilon .
$$

By the Sobolev embedding theorem this yields $\|\tilde{r}\|_{L^{\infty}}+\left\|\partial_{t} \tilde{r}\right\|_{L^{2}}=O(\varepsilon)$ and combining this with (3.6), we get (3.7) for $r=\gamma h_{1}(t-\phi)+\tilde{r}$.

\section{Proof of Theorem 1.1}

Assume that the hypotheses of Theorem 1.1 are fulfilled and denote by $u_{j}$ the scattering solutions related to $c_{j}, j=1,2$. Then, by Proposition 2.1

$$
\iint q(x) u_{1}(t, x, \theta) u_{2}(s-t, x, \theta) d x d t=0, \quad q:=c_{1}^{-2}-c_{2}^{-2} .
$$


for any $s \in \mathbf{R}, \theta \in S^{2}$. Let us apply now Proposition 3.1 and substitute $u_{j}, j=1,2$ in (4.1) by its singular expansion. We get

$$
\begin{aligned}
& -\int q \alpha_{1} \alpha_{2} \delta\left(s-\phi_{1}-\phi_{2}\right) d x \\
& =\int q\left[\alpha_{2} \beta_{1} h_{0}\left(s-\phi_{1}-\phi_{2}\right)+\alpha_{1} \beta_{2} h_{0}\left(s-\phi_{1}-\phi_{2}\right)+\alpha_{2} r_{1}\left(s-\phi_{2}\right)+\alpha_{1} r_{2}\left(s-\phi_{1}\right)\right] d x \\
& \quad+\iint q\left[\beta_{1} \beta_{2} h_{0}\left(t-\phi_{1}\right) h_{0}\left(s-t-\phi_{2}\right)+r_{1}(t) r_{2}(s-t)\right. \\
& \left.\quad+\beta_{1} h_{0}\left(t-\phi_{1}\right) r_{2}(s-t)+\beta_{2} h_{0}\left(s-t-\phi_{2}\right) r_{1}(t)\right] d x d t
\end{aligned}
$$

Here $r_{1}(t)=r_{1}(t, x, \theta), \phi_{1}=\phi_{1}(x, \theta)$ etc. Denote $\phi(x, \theta)=\phi_{1}(x, \theta)+\phi_{2}(x, \theta), a(x, \theta)=$ $\alpha_{1}(x, \theta)+\alpha_{2}(x, \theta)$. Since by Lemma 3.2, $\phi(x, \theta)$ is close to $2 x \cdot \theta$ and $a(x, \theta)$ is close to 1 , the left-hand side of (4.2) reminds us of the Radon transform $R q$ of $q$. Let us recall, that we have the following Parseval's equality for the Radon transform $\left\|\partial_{s} R f\right\|_{L^{2}\left(\mathbf{R} \times S^{2}\right)}=4 \pi\|f\|_{L^{2}}$. Bearing this in mind, let us differentiate (4.2) with respect to $s$.

$$
-\partial_{s} \int q a \delta(s-\phi) d x=I_{1}+I_{2}+I_{3}+I_{4}
$$

where

$$
\begin{aligned}
I_{1} & =\int q\left(\alpha_{2} \beta_{1}+\alpha_{1} \beta_{2}\right) \delta(s-\phi) d x \\
I_{2} & =\int q\left[\alpha_{2} \partial_{s} r_{1}\left(s-\phi_{2}\right)+\alpha_{1} \partial_{s} r_{2}\left(s-\phi_{1}\right)\right] d x \\
I_{3} & =\int q\left[\beta_{1} \beta_{2} h_{0}(s-\phi)+\beta_{1} r_{2}\left(s-\phi_{1}\right)+\beta_{2} r_{1}\left(s-\phi_{2}\right)\right] d x \\
I_{4} & =\iint q r_{1}(t) \partial_{s} r_{2}(s-t) d x d t .
\end{aligned}
$$

The left-hand side of (4.3) vanishes for $|s|>2 T$ (see Lemma 3.2 and (3.5)). Therefore, so does the right-hand side above, but this is not necessarily true for each term $I_{j}$. Let us estimate the norm in $L^{2}\left([-2 T, 2 T] \times S^{2}\right)$ of each term in (4.3). For the left-hand side in (4.3) we have

$$
\begin{aligned}
\| \partial_{s} \int q(x) a(x, \theta) \delta(s-\phi(x, \theta)) & d x \|_{L^{2}\left([-2 T, 2 T] \times S^{2}\right)} \\
& =(2 \pi)^{-1 / 2}\left\|k \int e^{i k \phi(x, \theta)} a(x, \theta) q(x) d x\right\|_{L^{2}\left(\mathbf{R}_{k} \times S_{\theta}^{2}\right)} .
\end{aligned}
$$

Let us extend $\phi(x, \xi), a(x, \theta)$ for $\xi \notin S^{2}$ by $\phi(x, \xi)=|\xi| \phi(x, \xi /|\xi|), a(x, \xi)=a(x, \xi /|\xi|)$. Then Lemma 3.2 implies

$$
\left|\partial_{x}^{\alpha} \partial_{\xi}^{\beta}(\phi(x, \xi)-2 x \cdot \xi)\right| \leq C_{1} \varepsilon|\xi|^{1-|\beta|} \text { for }|\alpha|+|\beta| \leq m, x \in B_{4 \rho}, \xi \neq 0 .
$$

Similarly, (3.6) implies

$$
\left|\partial_{x}^{\alpha} \partial_{\xi}^{\beta}(a(x, \xi)-1)\right| \leq C_{1} \varepsilon|\xi|^{-|\beta|} \quad \text { for }|\alpha|+|\beta| \leq m-2, x \in B_{4 \rho}, \xi \neq 0 .
$$


Since $q$ is real-valued, the square integral of the expression in the right-hand side of (4.4) over $\mathbf{R}_{k} \times S^{2}$ equals twice the square integral over $\mathbf{R}_{k}^{+} \times S_{\theta}^{2}$. Setting $\xi=k \theta, k>0, \theta \in S^{2}$, we obtain from (4.4)

$$
\left\|\partial_{s} \int q(x) a(x, \theta) \delta(s-\phi(x, \theta)) d x\right\|_{L^{2}\left([-2 T, 2 T] \times S^{2}\right)}=\sqrt{2}(2 \pi)^{-1 / 2}\|P q\|_{L^{2}\left(\mathbf{R}_{\xi}^{3}\right)},
$$

where

$$
(P q)(\xi)=\int e^{i \phi(x, \xi)} a(x, \xi) q(x) d x .
$$

Our plan is the following. First we will show that $C_{1}\|q\| \leq\|P q\| \leq C_{2}\|q\|$ with some $C_{1}>0$, $C_{2}>0$ independent of $\varepsilon$. Next we are going to estimate the norms in $L^{2}\left([-2 T, 2 T] \times S^{2}\right)$ of each term $I_{j}=I_{j}(s, \theta)$ in (4.3) and will show that $I_{j}=O(\varepsilon\|q\|), j=1,2,3,4$. Then (4.3), (4.7) would imply that $C_{1}\|q\| \leq\|P q\| \leq C \varepsilon\|q\|$, hence $q=0$.

Proposition 4.1 If $c_{j}, j=1,2$ satisfy (3.2) with $m=9$ and if $\varepsilon>0$ is sufficiently small, then $P: L^{2}\left(B_{\rho}\right) \rightarrow L^{2}\left(\mathbf{R}_{\xi}^{3}\right)$ is a bounded operator. Moreover there exist two constants $C_{1}>0$, $C_{2}>0$ independent of $\varepsilon$ (small enough), $c_{1}, c_{2}$, such that

$$
C_{1}\|f\| \leq\|P f\| \leq C_{2}\|f\| \quad \text { for any } f \in L^{2}\left(B_{\rho}\right) \text {. }
$$

Proof. We will show that the estimate above follows from the fact that $\phi=\phi_{1}+\phi_{2}$ is close to $2 x \cdot \theta$ (see Lemma 3.2) and $a$ is close to 1 (see 4.6). This does not necessarily implies that $P$ (see (4.8)) is close to the Fourier transfotm, but one can expect that $P^{*} P$ is close to $c I$ with some constant $c$. We have

$$
\left(P^{*} P f\right)(x)=\iint e^{-i(\phi(x, \xi)-\phi(y, \xi))} a(x, \xi) a(y, \xi) f(y) d y d \xi
$$

The phase function above admits the representation

$$
\phi(x, \xi)-\phi(y, \xi)=2(x-y) \cdot \eta(x, y, \xi)
$$

where

$$
\eta(x, y, \xi)=\frac{1}{2} \int_{0}^{1}\left(\nabla_{x} \phi\right)(x+t(x-y), \xi) d t .
$$

To prove (4.10) it is enough to apply the identity $g(1)-g(0)=\int_{0}^{1} g^{\prime}(t) d t$ to the function $g(t)=\phi(x+t(x-y))$. By Lemma 3.2, $\eta(x, y, \xi)$ belongs to $W^{m-1, \infty}$ and is homogeneous with respect to $\xi$ of order one. Moreover,

$$
\left|\partial_{x}^{\alpha} \partial_{y}^{\beta} \partial_{\xi}^{\gamma}(\eta(x, y, \xi)-\xi)\right| \leq C \varepsilon|\xi|^{1-|\gamma|} \text { for }|\alpha|+|\beta|+|\gamma| \leq m-1, x \in B_{4 \rho}, y \in B_{4 \rho}, \xi \neq 0 .
$$

The equation $\eta=\eta(x, y, \xi)$ can be solved for $\xi$ provided that $\varepsilon$ is sufficiently small. The Jacobian $J:=|D \eta / D \xi|$ satisfies the estimates

$$
\left|\partial_{x}^{\alpha} \partial_{y}^{\beta} \partial_{\xi}^{\gamma}(J(x, y, \xi)-1)\right| \leq C \varepsilon|\xi|^{-|\gamma|} \text { for }|\alpha|+|\beta|+|\gamma| \leq m-2, x \in B_{4 \rho}, y \in B_{4 \rho}, \xi \neq 0 .
$$


Let us perform the change of variables $\xi \rightarrow \eta$ in (4.9).

$$
P^{*} P f=\iint e^{-2 i(x-y) \cdot \eta} b(x, y, \eta) f(y) \tilde{J}(x, y, \eta) d y d \eta,
$$

where $\tilde{J}(x, y, \eta)=\left.J^{-1}(x, y, \xi)\right|_{\xi=\xi(x, y, \eta)}, b(x, y, \eta)=\left.a(x, \xi) a(y, \xi)\right|_{\xi=\xi(x, y, \eta)}$. The principal part of the integral above is

$$
\iint e^{-2 i(x-y) \cdot \eta} f(y) d y d \eta=\pi^{3} f
$$

so from (4.12) we get

$$
\left(P^{*} P-\pi^{3} I\right) f=\iint e^{-2 i(x-y) \cdot \eta} f(y)((b \tilde{J})(x, y, \eta)-1) d y d \eta .
$$

We are going to apply Theorem A.1 (see the Appendix below) to (4.13). By (4.11), (3.6),

$$
\left|\partial_{x}^{\alpha} \partial_{y}^{\beta}((b \tilde{J})(x, y, \eta)-1)\right| \leq C \varepsilon \quad \text { for }|\alpha|+|\beta| \leq m-2, x \in B_{4 \rho}, y \in B_{4 \rho}, \eta \neq 0 .
$$

Let us extend the operator $P^{*} P-\pi^{3} I$, defined a priori on $L^{2}\left(B_{\rho}\right)$ to an operator $Q$ in $L^{2}\left(\mathbf{R}^{3}\right)$ by (4.13) with $\tilde{J}-1$ replaced by $\chi(x)(\tilde{J}-1) \chi(y)$, where $\chi \in C_{0}^{\infty}$, supp $\chi \subset B_{2 \rho}, \chi=1$ on $B_{\rho}$. Then if $m-2=7$, Theorem A.1 yields $\|Q\|_{\mathcal{L}\left(L^{2}\left(\mathbf{R}^{3}\right)\right)} \leq C \varepsilon$, which implies

$$
\left\|P^{*} P-\pi^{3} I\right\|_{\mathcal{L}\left(L^{2}\left(B_{\rho}\right)\right)} \leq C \varepsilon .
$$

Thus, for any $f \in L^{2}\left(B_{\rho}\right)$ we have

$$
\left|\|P f\|^{2}-\pi^{3}\|f\|^{2}\right|=\left|\left(P^{*} P f-\pi^{3} f, f\right)\right| \leq C \varepsilon\|f\|^{2},
$$

and this completes the proof of Proposition 4.1 for $\varepsilon$ small enough.

We proceed now with estimating the norms of $I_{j}, j=1,2,3,4$ in $L^{2}\left([-2 T, 2 T] \times S^{2}\right)$. By (3.6) and (4.7) we get for $I_{1}$

$$
\begin{aligned}
\left\|I_{1}\right\|_{L^{2}\left([-2 T, 2 T] \times S^{2}\right)} & \leq C \varepsilon\left\|\int|q| \delta(s-\phi) d x\right\|_{L^{2}\left(\mathbf{R} \times S^{2}\right)} \\
& \leq C^{\prime} \varepsilon\left\|\partial_{s} \int|q| \delta(s-\phi) d x\right\|_{L^{2}\left(\mathbf{R} \times S^{2}\right)} \\
& \leq C^{\prime \prime}\left\|P_{0}|q|\right\| \leq C^{\prime \prime \prime}\|q\| .
\end{aligned}
$$

Here $P_{0}$ is the operator (4.8) with $a=1$. In order to prove (4.15), we have approximated $|q|$ with smooth functions and have used the fact that for any $f \in C^{1}(\mathbf{R})$ with $f=0$ outside some finite interval $[-a, a]$, we have $\|f\|_{L^{2}} \leq C(a)\left\|f^{\prime}\right\|_{L^{2}}$.

To estimate $I_{2}, I_{3}$ and $I_{4}$, observe that

$$
I_{2}+I_{3}+I_{4}=\int K(s, \theta, x) q(x) d x
$$


with

$$
\begin{aligned}
K=\alpha_{2} \partial_{s} r_{1}\left(s-\phi_{2}\right)+\alpha_{1} \partial_{s} r_{2}\left(s-\phi_{1}\right)+\beta_{1} \beta_{2} h_{0}(s-\phi) \\
\quad+\beta_{1} r_{2}\left(s-\phi_{1}\right)+\beta_{2} r_{1}\left(s-\phi_{2}\right)+\int_{-\rho}^{\rho+2 T} r_{1}(t) \partial_{s} r_{2}(s-t) d t
\end{aligned}
$$

When $|s|<2 T$ and $x \in B_{\rho}$, we have $\left|s-\phi_{2}\right| \leq 3 T,\left|s-\phi_{1}\right| \leq 3 T$. Next, in the integral term in (4.17) we have $|T|<3 T,-\rho \leq s-t \leq \rho+2 T<3 T$. Therefore, in (4.17) the argument of $r_{j}(t), j=1,2$ always belongs to the interval $|t| \leq 3 T$ thus we can apply Proposition 3.1 to get

$$
\int_{B_{\rho}} \int_{S^{2}} \int_{-2 T}^{2 T}|K(s, \theta, x)|^{2} d s d \theta d x \leq(C \varepsilon)^{2} .
$$

Therefore, by (4.16) we have

$$
\left\|I_{2}+I_{3}+I_{4}\right\|_{L^{2}\left([-2 T, 2 T] \times S^{2}\right)} \leq C \varepsilon\|q\| .
$$

Combining (4.3), (4.7), (4.15) and (4.18), we get

$$
\|P q\| \leq C \varepsilon\|q\|
$$

On the other hand, by Proposition 4.1 we conclude that

$$
C_{1}\|q\| \leq\|P q\|
$$

For $\varepsilon$ small enough (4.19) and (4.20) imply $q=0$. The proof of Theorem 1.1 is complete.

\section{A Appendix}

We prove here a theorem for the boundedness of $a(x, y, D)$ in $L^{2}\left(\mathbf{R}^{n}\right)$ if $a$ is smooth of finite order. Under the assumption that $a=a(x, \xi)$ is independent of $y$, Theorem 18.1.11' in $[\mathrm{H}]$ says that if $\int\left|\partial_{x}^{\alpha} a(x, \xi)\right| d x \leq M$ for all $\xi \in \mathbf{R}^{n}$ and for $|\alpha| \leq n+1$, then $\|a(x, D)\|_{\mathcal{L}\left(L^{2}\right)} \leq C M$ with $C>0$ an absolute constant. Following the proof of that theorem in $[\mathrm{H}]$, we obtain a generalization for amplitudes $a$ depending on $y$ as well.

Theorem A.1 Let $A$ be the operator

$$
A f=(2 \pi)^{-n} \iint e^{i(x-y) \cdot \xi} a(x, y, \xi) f(y) d y d \xi
$$

If

$$
\int\left|\partial_{x}^{\alpha} \partial_{y}^{\beta} a(x, y, \xi)\right| d x d y \leq M \quad \text { for }|\alpha|+|\beta| \leq 2 n+1, \xi \in \mathbf{R}^{n},
$$

then $\|A\|_{\mathcal{L}\left(L^{2}\right)} \leq C M$ with $C>0$ an absolute constant. 
Proof. We have

$$
A f=(2 \pi)^{-2 n} \iint e^{i x \cdot \xi} \tilde{a}(x, \xi-\zeta, \xi) \hat{f}(\zeta) d \zeta d \xi
$$

where $\tilde{a}(x, \zeta, \xi)=\int e^{-i \zeta \cdot y} a(x, y, \xi) d y$. Thus

$$
\begin{aligned}
\widehat{A f}(\eta):=\int e^{-i \eta \cdot x}(A f)(x) d x & =(2 \pi)^{-2 n} \iiint e^{-i x \cdot(\eta-\xi)} \tilde{a}(x, \xi-\zeta, \xi) \hat{f}(\zeta) d \zeta d \xi d x \\
& =(2 \pi)^{-2 n} \iint \tilde{\tilde{a}}(\eta-\xi, \xi-\zeta, \xi) \hat{f}(\zeta) d \zeta d \xi
\end{aligned}
$$

where $\tilde{\tilde{a}}(\eta, \zeta, \xi)=\int e^{-i \eta \cdot x} \tilde{a}(x, \zeta, \xi)=\int e^{-i(\eta \cdot x+\zeta \cdot y)} a(x, y, \xi) d x d y$. Therefore, $\widehat{A f}=B \hat{f}$, where $B$ is an integral operator with kernel

$$
b(\eta, \zeta)=(2 \pi)^{-2 n} \int \tilde{\tilde{a}}(\eta-\xi, \xi-\zeta, \xi) d \xi .
$$

We claim that $\int|b(\eta, \zeta)| d \eta \leq C M, \int|b(\eta, \zeta)| d \zeta \leq C M$. It is well known that this implies that $B$ is bounded with norm not exceeding $C M$.

$$
\int|b(\eta, \zeta)| d \eta \leq(2 \pi)^{-2 n} \iint|\tilde{\tilde{a}}(\eta-\xi, \xi-\zeta, \xi)| d \xi d \eta .
$$

The assumptions of the theorem imply $|\tilde{\tilde{a}}(\eta, \zeta, \xi)| \leq C M(1+|\eta|+|\zeta|)^{-2 n-1}$. Hence

$$
\begin{aligned}
\int|b(\eta, \zeta)| d \eta & \leq C^{\prime} M \iint(1+|\eta-\xi|+|\xi-\zeta|)^{-2 n-1} d \eta d \xi \\
& =C^{\prime} M \iint(1+|\eta|+|\xi-\zeta|)^{-2 n-1} d \eta d \xi \\
& =C^{\prime} M \iint(1+|\eta|+|\xi|)^{-2 n-1} d \eta d \xi \\
& =C^{\prime \prime} M<\infty .
\end{aligned}
$$

In the same way we treat $\int|b(\eta, \zeta)| d \zeta$.

\section{References}

[C-S] J. Cooper and W. Strauss, Scattering of waves by periodically moving bodies, J. Funct. Anal. 47(1982), 180-229.

[E-R] G. Eskin and J. Ralston, The inverse backscattering problem in 3 dimensions, Comm. Math. Phys. 124(1989), 169-215.

[G] V. Guillemin, Sojourn times and asymptotic properties of scattering matrix, Publ. Res. Inst. Math. Sci. 12(1976), 69-88.

[H] L. Hörmander, The Analysis of Linear Partial Differential Operators III, Springer, 1985.

[L-P] P. Lax And R. Phillips, Scattering Theory, Revised edition, Academic Press, 1989.

$[\mathrm{N}] \quad$ R. G. Novikov, Multidimensional inverse spectral problems for the equation $-\Delta \psi+(v(x)-$ $E u(x)) \psi=0$, Funct. Anal. and Appl. 22(1988), 263-272. 
[P] V. Petkov, Scattering Theory for Hyperbolic Operators, North-Holland, 1989.

[R-S] M. Reed And B. Simon, Methods of Modern Mathematical Physics, Vol. 3, Academic Press, 1979.

[St I] P. Stefanov, A uniqueness result for the inverse back-scattering problem, Inverse Probl. 6(1990), 1055-1064.

[St II] P. Stefanov, Generic uniqueness for two inverse problems in potential scattering, Comm. P.D.E. 17(1992), 55-68.

[S-U] J. Sylvester and G. Uhlmann, Global uniqueness for an inverse boundary value problem, Ann. Math. 125(1987), 153-169.

[U] G. Uhlmann, Inverse boundary value problems and applications, Astérisque 207(1992), $153-221$.

[V] B. R. VAinberg, Asymptotic Methods in Equations of Mathematical Physics, Gordon and Breach Sci. Publ., New York, 1988. 